\title{
Inteligencia emocional de los estudiantes de pregrado de las licenciaturas de enfermería
}

\author{
Emotional Intelligence of Undergraduate Nursing Students \\ Inteligência Emocional dos estudantes universitários de enfermagem
}

Como citar este artículo:

Torres Estrada Nallely, Sosa Rosas María del Pilar. Inteligencia emocional de los estudiantes de pregrado de las licenciaturas de enfermería. Revista Cuidarte. 2020;11(3):e993. http://dx.doi.org/10.15649/cuidarte.993

\section{Revista Cuidarte Rev Cuid. 2020; 11(3): e993 \\ doi) http://dx.doi.org/10.15649/cuidarte.993} E-ISSN: 2346-3414

(1) Nallely Torres Estrada ${ }^{1}$

(1) María del Pilar Sosa Rosas ${ }^{2}$

1 Servicio de oncología del Hospital Médica Sur, Ciudad de México. E-mail nalle. torres.e03@gmail.com

2 División de Estudios Profesionales de la Escuela Nacional de Enfermería y Obstetricia de la Universidad Nacional Autónoma de México, Ciudad de México. E-mail soosa99@yahoo.com Autor de Correspondencia.

\section{Resumen}

Introducción: La inteligencia emocional (IE) es una habilidad para percibir, asimilar y regular las propias emociones, saber actuar en el momento en que se presenta un problema que afecta la relación de los estudiantes, pasantes o enfermeras con los pacientes. Objetivo; Identificar niveles de IE de los estudiantes de las carreras de licenciatura en enfermería en 2017. Material y métodos: Estudio cuantitativo, descriptivo, transversal, a 90 estudiantes en Ciudad de México, se aplican 6 escalas (EIE-25, GQH-12, PANAS, ECE, MBI-SS y UWES-S), se utilizó la prueba estadística de Pearson y Fisher. Resultados: El 13.3\%(12) tienen adecuados niveles de IE, tienen satisfacción por el estudio el 100\%(12) de los que tienen adecuada IE, mientras que el $60.25 \%(47)$ en los de menor IE, con valor $p=0.007$. La edad, el semestre, sexo, el horario y la carrera reportaron $p>0.203$. Discusión: Resultados similares al de la Universidad de Veracruz México en que una menor IE repercute en la salud mostrando alteraciones en el estado de bienestar, lo descubierto en este estudio es que la enseñanza de la enfermería se ha centrado en las habilidades, competencias, aptitudes, la medición de lo intelectual más que en la IE. Conclusiones: La IE de los estudiantes de las licenciaturas en enfermería tiene una alta demanda emocional por lo que es conveniente realizar esfuerzos por las autoridades que implementan los planes de estudio para que se tengan estrategias que favorezcan el desarrollo de la IE y no únicamente el desarrollo intelectual.

Palabras clave: Inteligencia Emocional; Estudiantes de Enfermería; Competencia Emocional.

Recibido: septiembre 13 de 2019 Aceptado: junio 9 de 2020

$\square *$ Correspondencia María del Pilar Sosa Rosas

Publicado: septiembre 1 de 2020 


\section{Emotional Intelligence of Undergraduate Nursing Students}

\section{Abstract}

Introduction: Emotional Intelligence (EI) is the ability to perceive, assimilate and regulate one's own emotions, as well as to know how to act when there is a problem affecting the relationship of students, interns or nurses with patients. Objective: To identify El levels in undergraduate nursing students in 2017. Materials and Methods: A descriptive quantitative cross-sectional study was conducted with 90 undergraduate nursing students in Mexico City. Six scales were applied (EIS-25, GHQ-12, PANAS, EES, MBI-SS and UWES-S). Pearson's chi-squared test and Fisher's exact test were also applied. Results: $13.3 \%(12)$ have adequate levels of El, from which $100 \%(12)$ are satisfied with their studies, compared to $60.25 \%(47)$ in those with lower El ( $p=0.007)$. Age, academic term, gender, class hours and degree reported a p-value of $>0.203$. Discussion: Similar results were obtained to those of the University of Veracruz, Mexico which revealed that a lower El has an effect on health and alterations in emotional well-being. The study found out that nursing education has traditionally focused on skills, competencies, aptitudes and intellectual measurement rather than El. Conclusions: El in undergraduate nursing students has high emotional demands so authorities should make efforts to implement study programs that include strategies promoting El development more than just intellectual development.

Key words: Emotional Intelligence; Students, Nursing; Emotional Competence.

\section{Inteligência Emocional dos estudantes universitários de enfermagem}

\section{Resumo}

Introdução: A Inteligência Emocional (IE) é a capacidade de perceber, assimilar e regular as próprias emoções, bem como de saber como agir quando há um problema que afeta o relacionamento de estudantes, estagiários ou enfermeiras com os pacientes. Objetivo: Identificar os níveis de IE em estudantes universitários de enfermagem em 2017. Materiais e métodos: Um estudo descritivo quantitativo transversal foi realizado com 90 estudantes universitários de enfermagem na Cidade do México. Seis escalas foram aplicadas (EIE-25, GHQ-12, PANAS, ECE, MBI-SS e UWES-S). Também foram aplicados o teste de chi-quadrado de Pearson e o teste exato de Fisher. Resultados: 13,3\% (12) têm níveis adequados de El, dos quais 100\% (12) estão satisfeitos com seus estudos, em comparação com $60,25 \%(47)$ nos que têm IE mais baixo ( $p=0,007)$. Idade, semestre acadêmico, sexo, horas de aula e estudos de graduação informaram um p-valor de >0,203. Discussão: Resultados similares aos da Universidade de Veracruz, México, que revelaram que uma IE menor tem um efeito sobre a saúde e alterações no bem-estar emocional. $O$ estudo descobriu que a educação em enfermagem tem tradicionalmente se concentrado em habilidades, competências, aptidões e medição intelectual em vez de IE. Conclusões: A IE em estudantes universitários de enfermagem tem altas exigências emocionais, portanto, as autoridades devem fazer esforços para implementar programas de estudo que incluam estratégias que promovam o desenvolvimento da IE mais do que apenas o desenvolvimento intelectual.

Palavras chave: Inteligência Emocional; Estudantes de Enfermagem; Competência Emocional. 


\section{Introducción}

La Salud en el ser humano es comprendida más allá del reduccionismo biológico, como una red compleja de lo biológico, psicológico, social, cultural, económico y espiritual, lo que nos permite investigar lo psicológico, que es donde se ubican con mayor frecuencia las "emociones", que son una característica del ser humano, que tiene que conocer y manejar el profesional de enfermería, por el contacto directo con las personas enfermas durante el cuidado que otorga al hombre. Las emociones que experimentan los seres humanos juegan un papel fundamental en la dinámica de todos los fenómenos sociales, hace falta investigar más sobre ellas, como algo que influye para la conservación de la salud mental; de ahí que es importante acercarse al proceso de enseñanza-aprendizaje de los estudiantes de pregrado de enfermería porque es donde se comienzan a adquirir habilidades y destrezas para el cuidado del ser humano en lo psicológico (emociones).

Los profesionales de enfermería están constantemente en contacto con seres humanos, quienes son vulnerables al tener diferentes tipos de enfermedades. El contacto con ellos, se inicia durante las prácticas de enfermería cuando se es estudiante de pregrado, en donde se trata de propiciar una práctica sustentada en el dominio del cuidado basado en el conocimiento científico, las tecnologías y las prácticas terapéuticas complementarias con una actitud empática, flexible, crítica y solidaria a fin de brindar un cuidado holístico a la persona ${ }^{1}$, independientemente de que se mencione la empatía, en pocas ocasiones se toman en cuenta las emociones de los estudiantes, quienes comienzan a cuidar a los seres humanos no sólo en lo social y en lo biológico sino también en las emociones, llegando incluso en algunas ocasiones a vivir, sentir y compartir conjuntamente con el ser humano que cuida las emociones que tiene durante su estancia en una institución hospitalaria ${ }^{2}$, de

En pocas ocasiones se toman en cuenta las emociones de los estudiantes, quienes comienzan a cuidar a los seres humanos no sólo en lo social y en lo biológico sino también en las emociones, llegando incluso en algunas ocasiones a vivir, sentir y compartir conjuntamente con el ser humano que cuida. ahí que es importante hablar de inteligencia emocional (IE) de los estudiantes de pregrado en enfermería.

El aprendizaje que gira en torno a estudios formales se relaciona con la formación académica, en cada uno de los niveles de estudio de primaria, secundaria, bachillerato, licenciatura, maestría y doctorado, con respecto a los planes de estudio sé trabaja alrededor de la obtención de conocimiento a través del coeficiente intelectual que de la inteligencia emocional. La estructura y diseño de las universidades, tradicionalmente han respondido a un perfil de estudiante maduro, responsable e independiente; en otras palabras las universidades han sido planeadas y creadas para alumnos adultos, con capacidad de auto determinarse e invertir un esfuerzo y tiempo para el logro de sus metas profesionales, pero parece que en los últimos años, las universidades no pueden cumplir con su misión, ya que el nivel de reprobación, bajo rendimiento académico y deserción universitaria es muy alta ${ }^{3}$.

En el área de enfermería, la IE es un concepto clave para la comunicación con los seres humanos y la organización de las destrezas interpersonales en el ámbito de la profesión. La IE en estudiantes de enfermería ayuda en la relación estudiante-paciente, estudiante-enfermera(ro), estudiante-médico(ca), estudiante-profesor(ra), enfermera(ro) y aspectos relacionados con la calidad del cuidado, la satisfacción del paciente, el rendimiento, el nivel de implicación, la satisfacción profesional, y finalmente en el entrenamiento y desarrollo de las habilidades de comunicación clínica ${ }^{4}$. 
Tras diversos estudios se ha comprobado que la IE refleja la fusión de la conciencia y conocimiento sobre sí mismo, la capacidad para manejar las emociones personales desarrolla la comprensión para entender los sentimientos de otros y la habilidad social que permite la interacción entre otros, como aspecto fundamental de la persona ${ }^{3}$. Este término apareció varias veces en la literatura, sin embargo la IE tiene sus raíces en el concepto de inteligencia social identificado por primera vez, por Thorndike en 1920, quien define la inteligencia como la capacidad de entender, gestionar y actuar con prudencia en las relaciones humanas 5 . P. Salovey y J.D. Mayer (1990), definen la IE como aquella parte de la inteligencia social que conlleva la capacidad para controlar nuestras emociones y las de los demás, discriminar entre ellas y usar dicha información para guiar nuestros pensamientos y comportamientos. A partir de esta fecha se considera como tema de interés general por parte de la sociedad, la publicación de Emotional Intelligence de Daniel Goleman (1995) significa una difusión sin precedentes de un concepto que hasta entonces había pasado desapercibido para el gran público ${ }^{6}$.

A partir de la gran variedad de constructos de la IE, diferentes autores han aportado su propuesta de definición a través de diferentes modelos teóricos. Cabe señalar que existen tres concepciones: 1) una que menciona que la IE es un conjunto de habilidades y capacidad, 2) una característica de personalidad y 3) Modelos que asumen a la IE como un conjunto de habilidades emocionales y personales ${ }^{7}$. Siguiendo la propuesta de Mayer, se puede distinguir entre modelos de la inteligencia emocional basados en el procesamiento de la información emocional, como el propio modelo de habilidades y modelo mixto caracterizados por contemplar las habilidades emocionales como los rasgos de personalidad. Dentro del modelo mixto se situarían la propuesta de Goleman (modelo de las competencias emocionales) y la de Reuven Bar-On (modelo de la inteligencia emocional y social) ${ }^{8}$.

La IE es un concepto clave para organizar las destrezas y/o habilidades y capacidades interpersonales de comunicación del estudiante o profesional de enfermería con el ser humano que cuida, es muy importante identificar la IE de los estudiantes de pregrado en enfermería durante su formación académica para fomentar la habilidad de saber cómo actuar emocionalmente.

En el nivel superior nunca se han tomado en cuenta lo que siente el estudiante, lo importante para este nivel es que el estudiante acredite las materias y que obtenga buenas calificaciones para obtener un título que le permita trabajar, diversos autores mencionan que la IE refleja el $80 \%$ del éxito académico y/o laboral y solo el $20 \%$ involucra el coeficiente intelectual, siendo la capacidad para conceptualizar las emociones personales, desarrollar la comprensión para entender los sentimientos de otros y la habilidad social que permite la interacción entre otros, como aspectos fundamentales de la persona ${ }^{9-10}$.

Independientemente de las habilidades y destrezas de cada estudiante que puede adquirir durante su formación académica de pregrado en enfermería, tienen momentos de duda, desinterés, miedo, frustración, estrés, angustia, tristeza, sorpresa, ansiedad, enojo, uno que otro caso con depresión y cansancio emocional por diversos factores entre ellos: las calificaciones, sobre carga de estudio, satisfacción o insatisfacción con la carrera, semestres de los cursos anteriores, mantener un promedio, horas de estudio personal, diferentes cuestiones familiares y sociodemográficas con los que cada alumno cuenta, incorporando a ello a los profesores que juegan un papel fundamental en la vida académica del estudiante ${ }^{11}$.

El profesor de enfermería se considera primordial en la adquisición de nuevos conocimientos, son el medio para alcanzar los objetivos académicos, reforzar valores, desarrollo de conciencia crítica y reflexiva, pero principalmente, fomentar una actitud propositiva a fin que los estudiantes tengan una plena conciencia de los distintos hechos que sucedan consigo mismo, sin 
embargo, muchos de ellos tienen excelentes conocimientos dentro de su área de estudio, pero no todos tienen las habilidades pedagógicas para establecer y conservar relaciones interpersonales significativas, necesarias para tener una relación de confianza entre profesor y alumno ${ }^{12-13}$.

En la revisión de la literatura se buscó información sobre IE en estudiantes de pregrado de enfermería, en dicha revisión no se encontraron muchos datos relacionados, con respecto a México, no hay muchos estudios de IE realizados con estudiantes de pregrado de enfermería durante su formación académica, sin embargo, se encontró un estudio similar dentro de la República Mexicana y estudios relacionados en otros países que hacen referencia a alteraciones en los estados emocionales de los estudiantes de pregrado de enfermería. Estas investigaciones indicaron que la falta de IE es una problemática que se presenta en el contexto en que aprende el estudiante, que es necesario ponerle atención porque el desarrollo de la IE ayuda al progreso personal de los estudiantes de enfermería, en el sentido de cuidar de su persona en primera instancia para que posteriormente puedan cuidar bien a los otros ${ }^{14}$.

Por todo lo mencionado, se considera transcendente estudiar la IE durante la formación académica de los estudiantes de las licenciaturas en enfermería, con el propósito de identificar niveles de IE de los estudiantes de las carreras de licenciatura en enfermería.

\section{Material y métodos}

Estudio cuantitativo, de tipo descriptivo de corte transversal, no experimental. Se aplica un instrumento con 6 escalas validadas, del 1 de septiembre al 10 de diciembre del 2017, a los estudiantes de la licenciatura de enfermería y licenciatura de enfermería y obstetricia de los turnos matutino y vespertino, en los semestres 1ro., 2do., 7 mo., y 8vo., bajo la declaración de Helsinki del año 2000 y de la Ley General de Salud, previo consentimiento informado, se les explica los objetivos de la investigación de manera escrita y verbal, se les informa de los beneficios que obtienen al participar en el llenado del cuestionario y la importancia de la misma, se selecciona una muestra siguiendo los criterios de conveniencia, accesibilidad y disponibilidad de los participantes.

Se utiliza el muestreo por conveniencia, conformado por 90 estudiantes de enfermería de pregrado; 65 son mujeres y 25 hombres, 35 del turno matutino, 55 del turno vespertino, 51 de la licenciatura en enfermería (LE), 39 de la licenciatura en enfermería y obstetricia (LEO), de los cuales 39 son de 1 er y 2 do semestre, 51 de $7 \mathrm{mo}$. y 8vo. Semestre, con criterios de inclusión de estar inscrito en la institución educativa, en los turnos matutino y vespertino, asistencia en el momento de aplicación del instrumento, aceptación de participar voluntariamente, los criterios de exclusión son no estar inscritos en la institución educativa, no encontrarse en el momento de aplicación del instrumento, no querer participar y los criterios de eliminación es que no llenaran correctamente el instrumento.

La inteligencia emocional conceptualiza el control de las emociones, el control de los propios impulsos, el control ante situaciones adversas, la frustración ante las tareas cotidianas, síntomas emocionales, bienestar psicológico, sentimientos, presencias de estados depresivos, ansiedad, estrés, cansancio emocional de las personas, de ahí la importancia de la aplicación de los siguientes instrumentos:

Escala de Inteligencia Emocional (EIE-25) versión inspirada en los 5 componentes de Goleman con 0.815 de fiabilidad ${ }^{15}$ son 25 reactivos, la versión utilizada comprende cinco componentes (5C-IE); 1. Conciencia emocional, 2. Control emocional, 3. Empatía, 4. Motivación y 5. Habilidades sociales, escala tipo Likert de cinco ítems, la puntuación es a través de una codificación de 
ítems con puntuaciones 1-2-3-4-5, siendo: $1=$ Nada de acuerdo, 2= Algo de acuerdo, 3= Bastante de acuerdo, 4= Muy de acuerdo y $5=$ Totalmente de acuerdo. Cada componente proporciona información sobre: Conciencia emocional: conciencia de los estados emocionales. (ítem 1, 6, 11, 16 y 21, Control emocional: muestra el grado de capacidad de la persona para manejar y controlar las emociones.( Ítem 2, 7, 12, 17 y 22), La empatía: se refiere al grado en que una persona sabe entender y comprender los sentimientos y las emociones de los demás (ítem 3, 8, 13, 18 y 23), Motivación: evalúa el grado de expectativas que la persona desarrolla para conseguir sus objetivos.(ítem 4, 9, 14, 19 y 24), Habilidades sociales: incluye comunicación o desarrollo de habilidades sociales. (Ítem 5, 10, 15, 20, 25). La puntuación total, es la suma de las dimensiones, en esta investigación la puntuación total es de 125 puntos, el punto de corte es de 100 puntos, por lo tanto una puntuación menor a 100, nos indica que la persona tiene menor tendencia para su rendimiento de la competencia emocional con un déficit en el manejo de la Inteligencia emocional. Una puntuación mayor a 100 nos refleja a personas con un adecuado control de impulsos que buscan solucionar los problemas que enfrentan en su vida cotidiana, de una manera convencional².

(GQH-12) Escala de Salud General de Goldberg, con 0.791 de fiabilidad ${ }^{16}$ se centra en dos tipos de problemas, la dificultad auto - percibida y la aparición de fenómenos de naturaleza angustiosa para la persona, instrumento para evaluar síntomas emocionales, bienestar psicológico o trastornos mentales, se utiliza una versión de12 ítems por su brevedad y rápida administración, escala tipo Likert con cuatro anclajes; funciones intelectuales y fisiológicas (ítems 1 y 2), recogen puntuaciones respecto a la evaluación que los individuos hacen de su estado de salud (ítems 3, 4, 5, 9 y 12), mide la auto-valoración en el establecimiento y alcance de las metas (ítems 10 y 11). Información referente al afrontamiento de las dificultades (ítem 6). Se emplea la forma de puntuación más conocida a través de una codificación de ítems con las puntuaciones 0-12-3, una puntuación mayor indica mayor severidad psicológico y viceversa. La escala GHQ-12 muestra que está compuesta por síntomas de ansiedad y depresivos, como los clasifican en la actualidad la Organización Mundial de la Salud y la Asociación Psiquiátrica Americana ${ }^{16}$. La puntuación total se calcula sumando las calificaciones obtenidas en todos los enunciados de la escala. Según este método, el valor máximo que se puede obtener en la escala es de 36 puntos y el mínimo de cero puntos. El modo en el que se distribuyan las puntuaciones indicará mayor o menor salud mental y bienestar psicológico².

Escala de Afecto Positivo y Negativo (PANAS) versión española de Sandin y Cols con un nivel de fiabilidad de PA: 0.77 y NA: $0.91^{17}$, evalúa dos factores con 20 adjetivos que describen sentimientos y emociones, la mitad de los adjetivos evalúan afecto positivo y la otra mitad evalúa afecto negativo, escala tipo Likert de cinco opciones de respuesta de acuerdo al grado en que las experimenta; 1 = Nada o casi nada, 2= Poco, 3= A medias, 4= Bastante y 5= Mucho o muchísimo.

En el primer apartado se evalúa la presencia de los afectos "en las últimas semanas" (afecto como estado) y en el segundo apartado se les evalúa "generalmente" (afecto como rasgo), de la puntuación ya que se abarca el nivel de afecto negativo en pasado y presente. El rango de la escala PANAS corresponde a la puntuación total de 200 puntos, siendo el punto de corte de 80 y la puntuación más baja es de 40, por lo tanto las personas con bajo nivel de afecto positivo muestran mayor inclinación a experimentar sentimientos de tristeza y depresión, mientras que las personas con altos niveles de afecto negativo frecuentemente experimentan emociones tales como la ansiedad, culpa u hostilidad, la escala PANAS ha demostrado ser una medida válida y confiable para evaluar la presencia de estados depresivos, rasgos de personalidad, ansiedad, estrés y adaptación en población clínica y normal, de adolescentes, adultos y adultos mayores ${ }^{17}$. 
Escala de Cansancio Emocional para Universitarios (ECE) de Ramos, Magan; Moran con 0.809 de fiabilidad ${ }^{11}$. Escala autoinforme de 12 ítems de tipo Likert de 5 puntos donde: $1=$ Raras veces, $2=$ Pocas veces, $3=$ Algunas veces, $4=$ Con frecuencia y $5=$ Siempre. Los diez primeros ítems poseen una estructura unidimensional y hacen referencia a la escala "Cansancio Emocional" (CE), que evalúan situaciones relacionadas con el síndrome de burnout. Los dos últimos ítems integran dos escalas, una cada uno: la escala de "Satisfacción con el estudio" (SE): "Disfruto estudiando porque me gusta lo que estudio", y la escala de "Autoestima" (Au): "Me veo a mí mismo como alguien que tiene alta autoestima", durante los últimos doce meses de vida académica. La puntuación de la escala ECE su calificación oscila entre los 10 puntos mínimos y los 50 como máximos. El punto de corte es de 25 puntos, mayor a 25 puntos indica cansancio emocional evalúa situaciones relacionadas con el factor de Burnout y viceversa, para las escalas de "SE" y "Au" su puntuación máxima es de 5, puntuación mínima de 1 y punto de corte de 3 . Para la escala de "SE" menor a 3 puntos indica insatisfacción con el estudio y viceversa. En el caso de la escala de "Au" menor a 3 puntos indica bajo autoestima y viceversa"

Cuestionario de Estrés para Estudiantes (MBI-SS) de Schaufeli, Salonava, González-Roma y Bekker con 0.730 de fiabilidad ${ }^{18-19}$ mide el "estrés en estudiantes", esta escala se conforma de 3 dimensiones, cada una se encarga de; agotamiento (por las demandas de estudio); calcula la percepción de encontrarse física, mental y emocionalmente exhausto y con una sensación de no poder más de sí mismo frente a las actividades académicas (ítem 1, 2, 10, 11 y 12), cinismo: Mide la actitud negativa del estudiante frente a sus estudios, evidenciada por la autocrítica, pérdida de interés y de valor frente al estudio (ítem 3, 8, 13 y 14) eficacia académica: Evalúa la percepción del universitario sobre la competencia en sus estudios (ítem 4,5, 6, 7, 9 y 15).

Todos los ítems de cada una de estas tres subescalas se puntúan en una escala de frecuencia de 7 puntos, que va desde 0 (nunca) a 6 (siempre). Cinco ítems evalúan agotamiento, cuatro evalúan cinismo y seis ítems evalúan autoeficacia académica, para un total de 15 reactivos la puntuación total es de 90 puntos, su puntuación de corte es de 45 puntos.

El estudiante está más quemado (estresado) cuando manifiesta altas puntuaciones en agotamiento y cinismo y bajas puntuaciones en su creencia de eficacia académica. Esto quiere decir que menor a 45 puntos presenta eficacia académica y mayor a 45 puntos tiene mayor agotamiento y cinismo manifestando mayor estrés, bajo compromiso e interés académico ${ }^{18-20}$. Escala Engagement para Estudiantes (UWES-S) de Schaufeli y Bakker, traducido al español por Salanova con 0.9008 de fiabilidad ${ }^{21}$, escala de 17 ítems para medir tres dimensiones; vigor (6 ítems): Mide la energía y la resistencia mental mientras se está estudiando, el deseo de invertir esfuerzo, tiempo, persistencia en el estudio, incluso cuando aparecen obstáculos y barreras, ítem de esta dimensión: 5, 8, 9, 12, 15 y 16, absorción (6 ítems); evalúa un estado agradable de inmersión total en el trabajo, el individuo es incapaz de separarse a pesar de que ha pasado mucho tiempo, Ítem de esta dimensión: 1, 2, 3, 10, 11 y 13, dedicación (5 ítems): Evalúa el entusiasmo, orgullo, inspiración y reto relacionado con los estudios, Ítem de esta dimensión; 4, 6, 7, 14 y 17, se aplica con escala de tipo Likert con 7 posiciones; 0 significa totalmente en desacuerdo y 6 totalmente de acuerdo con la afirmación reflejada en el ítem. La puntuación total es de 102, su puntuación de corte es de 51, esto nos indica que mayor a 51 puntos refleja elevados niveles de engagement (mayor compromiso e interés) y menor a 51 puntos presentan menor compromiso e interés académico por insatisfacción de realizar actividades académicas ${ }^{21}$.

Para la elaboración de los resultados del estudio se utiliza el programa Excel que permitió construir una base de datos de los instrumentos aplicados lo que dio la oportunidad de identificar la manera en que se podría agrupar la información, posteriormente se ejecuta el programa 
SPSS 23 para agrupar la información obtenida mediante la estadística descriptiva a través de los cuestionarios ejecutando la distribución de frecuencias, medidas de tendencia central (media, mediana y moda), medidas de variabilidad (rango, desviación estándar y varianza). En la identificación de diferencias entre los grupos de adecuada IE y Menor IE se utilizó la prueba estadística de Fisher, se tomó como punto de corte un valor $\mathrm{p}<0.005$ para determinar diferencias estadísticamente significativas, el uso de la información obtenida se manejó de forma anónima y confidencial, con el fin de evitar exhibir a los estudiantes. Los resultados obtenidos fueron manejados con profesionalismo y cuidado de la imagen institucional.

\section{Resultados}

Se aplicó un instrumento con 6 escalas validadas a 90 estudiantes de la Licenciatura en Enfermería y Licenciatura en Enfermería y Obstetricia, en la Tabla 1 se muestra una comparación de niveles de IE según características sociodemográficas y educativas, se identificó que no hay diferencias estadísticamente significativas (valor $p<0.05$ ); la más relevante fue el horario donde en los de menor IE el 35.89\%(28) tienen horario matutino y los de adecuada IE el 58.33\%(7), con un valor $p=0.203$. En relación a la edad se identificó que los mayores de 26 años tienen menor IE representaron el $14.11 \%(11)$, mientras en los de adecuada IE fueron el 25\%(3).

La variable de sexo, semestre y carrera reportaron un valor $\mathrm{p}$ mayor a 0.5 y las diferencias ante los grupos fueron menores.

Tabla 1. Comparación de niveles de IE según características sociodemográficas y educativas

\begin{tabular}{|c|c|c|c|c|}
\hline Variable & $\begin{array}{l}\text { Todos } \\
\% \text { (n) }\end{array}$ & $\begin{array}{l}\text { Menor IE } \\
\mathbf{8 6 . 6}(78)\end{array}$ & $\begin{array}{c}\text { Adecuada IE } \\
13.3(12)\end{array}$ & Valor $p$ \\
\hline Sexo \%(n) & & & & 1.00 \\
\hline Femenino & $72.2(65)$ & $71.8(56)$ & $75.0(9)$ & \\
\hline Masculino & $27.8(25)$ & $28.2(22)$ & 25.0(3) & \\
\hline Semestre & & & & 0.542 \\
\hline $1 / 2$ semestre & $43.3(39)$ & $44.9(35)$ & $33.3(4)$ & \\
\hline $7 / 8$ semestre & $56.7(51)$ & $55.1(43)$ & $66.7(8)$ & \\
\hline Edad & & & & 0.497 \\
\hline $18-20$ años & $45.6(41)$ & $46.2(36)$ & $41.7(5)$ & \\
\hline $21-23$ años & $27.8(25)$ & $28.2(22)$ & $25.0(3)$ & \\
\hline $24-26$ años & $11.1(10)$ & $11.5(9)$ & $8.3(1)$ & \\
\hline$>26$ años & $15.6(14)$ & $14.1(11)$ & $25.0(3)$ & \\
\hline Horario & & & & 0.203 \\
\hline Matutino & $38.9(35)$ & $35.89(28)$ & $58.33(7)$ & \\
\hline Vespertino & $61.1(55)$ & $64.10(50)$ & $41.66(5)$ & \\
\hline Carrera & & & & 0.542 \\
\hline Licenciatura en Enfermería & $56.7(51)$ & $55.1(43)$ & $66.7(8)$ & \\
\hline $\begin{array}{l}\text { Licenciatura en Enfermería } \\
\text { y Obstetricia }\end{array}$ & $43.3(39)$ & $44.9(35)$ & $33.3(4)$ & \\
\hline
\end{tabular}

IE: Inteligencia emocional.

Fuente: Resultado de instrumentos aplicados del 1 de septiembre al 10 de diciembre del 2017 en una Universidad de la Ciudad de México.

En la Tabla 2 se puede destacar que las escalas psicométricas aplicadas a los estudiantes revelaron que significativamente tiene relación con la conceptualización de las emociones que experimenta el alumno de las licenciaturas de enfermería durante su formación académica, la escala más relevante fue la de Satisfacción con el Estudio (SE) donde se identificó satisfacción con el estudio en el 100\%(12) de los que tienen adecuada IE y en el 60.25\%(47) de los que presentan menores puntajes de IE, con un valor $p=0.007$. Por otro lado es relevante encontrar que en 
la escala de PANAS en la dimensión de afectos negativos donde tan solo el $1.1 \%$ no tienen alteraciones en este, el cual está en grupo de los de adecuada $\mathrm{IE}$, con valor $\mathrm{p}=0.133$, también, se identifica que el $80 \%$ (72) tiene tristeza y depresión.

En la escala de GQH-12, se identifica que el 43.3\%(39) presentan puntajes de malestar psicológico, este porcentaje en los de adecuada IE fue de $41.66 \%$ (5) y en los de menor IE fue de $43.58 \%(34)$ y no se identificó diferencia entre los grupos con valor $p=1.0$.

En la escala ECE con valor de $p=0.725$ se identificó que el $77.7 \%$ (70) tiene cansancio emocional en relación con el Síndrome de Burnout como síntoma, del cual el $78.20 \%$ (61) manifiesta menor IE y el $75.00 \%$ (9) adecuada IE.

Las escalas Au, MBI-SS y UWES-S, reportan \%(n) menor en cuanto resultados negativos.

Tabla 2. Comparación de niveles de IE según escalas psicométricas

\begin{tabular}{|c|c|c|c|c|}
\hline Variable & $\begin{array}{c}\text { Todos \% (n) } \\
100(90)\end{array}$ & $\begin{array}{l}\text { Menor IE } \\
86.6(78)\end{array}$ & $\begin{array}{c}\text { Adecuada IE } \\
13.3(12)\end{array}$ & $\begin{array}{c}\text { Valor } \\
\mathbf{p}\end{array}$ \\
\hline \multicolumn{4}{|c|}{ GQH = 12 (Escala de Salud General de Goldberg) } & 1.00 \\
\hline Malestar psicológico & 43.3(39) & $43.58(34)$ & $41.66(5)$ & \\
\hline Bienestar psicológico & $56.6(51)$ & $56.41(44)$ & $58.33(7)$ & \\
\hline \multicolumn{4}{|c|}{ PANAS ( Escala de Afecto Positivo y Negativo) } & 0.700 \\
\hline \multicolumn{5}{|l|}{ *PA (Afecto Positivo) } \\
\hline Tristeza y depresión & $80(72)$ & $80.76(63)$ & $75.00(9)$ & \\
\hline Afecto positivo & 20(18) & $19.23(15)$ & $25.00(3)$ & \\
\hline *NA (Afecto Negativo) & & & & 0.133 \\
\hline Estado de ansiedad, culpa y hostilidad & $98.8(89)$ & $100.00(78)$ & $91.66(11)$ & \\
\hline Sin alteración & $1.1(1)$ & $0.00(0)$ & $8.33(1)$ & \\
\hline \multicolumn{4}{|c|}{ ECE ( Escala de Cansancio Emocional para Universitarios) } & 0.725 \\
\hline $\begin{array}{l}\text { Tiene cansancio emocional en relación } \\
\text { con el Síndrome de Burnout como } \\
\text { síntoma. }\end{array}$ & $77.7(70)$ & $78.20(61)$ & $75.00(9)$ & \\
\hline No tiene Cansancio emocional & $22.2(20)$ & $21.79(17)$ & $25.00(3)$ & \\
\hline \multicolumn{4}{|l|}{ *SE (Satisfacción con los Estudios) } & 0.007 \\
\hline Insatisfacción con los estudios & $34.4(31)$ & $39.74(31)$ & $0.00(0)$ & \\
\hline Satisfacción con los estudios & $65.5(59)$ & $60.25(47)$ & $100.00(12)$ & \\
\hline \multicolumn{4}{|l|}{ *Au (Autoestima) } & 0.349 \\
\hline Baja autoestima & $40(36)$ & $42.30(33)$ & $25.00(3)$ & \\
\hline Adecuada autoestima & $60(54)$ & $57.69(45)$ & $75.00(9)$ & \\
\hline \multicolumn{4}{|c|}{ MBI - SS ( Cuestionario de Estrés para Estudiantes) } & 0.739 \\
\hline $\begin{array}{l}\text { Agotamiento y cinismo manifestando } \\
\text { mayor estrés, bajo compromiso e } \\
\text { Interés académico }\end{array}$ & $28.8(26)$ & $28.20(22)$ & $33.33(4)$ & \\
\hline Eficacia académica & $71.7(64)$ & $71.79(56)$ & $66.66(8)$ & \\
\hline \multicolumn{4}{|c|}{ UWES - S (Escala Engagement para Estudiantes) } & 0.205 \\
\hline $\begin{array}{l}\text { Menor compromiso e interés } \\
\text { académico por insatisfacción de } \\
\text { realizar actividades académicas }\end{array}$ & $16.6(15)$ & 19.23(15) & $0.00(0)$ & \\
\hline Compromiso e interés académico & $83.3(75)$ & $80.76(63)$ & $100.00(12)$ & \\
\hline
\end{tabular}

IE: Inteligencia emocional.

Fuente: Resultado de instrumentos aplicados del 1 de septiembre al 10 de diciembre del 2017 en una Universidad de la Ciudad de México.
En la escala de PANAS en la dimensión de afectos negativos donde tan solo el $1.1 \%(1)$ no tienen alteraciones en este, el cual está en el grupo de los de adecuada IE, con valor $\mathrm{p}=0.133$, también, se identifica que el $80 \%$ (72) tiene tristeza y depresión. 


\section{Discusión}

Contrastando nuestros resultados con el artículo de la Universidad de Veracruz en México ${ }^{3}$, hay una gran semejanza a pesar de la incompatibilidad con el instrumento, sin embargo ambos instrumentos se basan en el mismo objetivo conocer la Inteligencia emocional en estudiantes, por esta razón nos permite identificar factores similares por lo que podemos decir que una menor IE repercute en la salud mostrando alteraciones en el estado de bienestar de los estudiantes de pregrado. Ellos utilizan la versión TMMS-24 teniendo como resultado porcentajes similares tales como "el $56.7 \%$ de los estudiantes presenta menor IE y $43.3 \%$, presenta adecuada IE"3. En el presente estudio se utiliza la versión EIE-25, teniendo como resultado $86.6 \%$ con menor IE y $13.3 \%$ con adecuada IE, estadísticamente, no hay diferencia entre el nivel de IE en cuanto a las variables sexo, semestre, edad, horario y carrera, datos que son similares en el artículo antes mencionado, se observa que una menor inteligencia emocional repercute en la salud, mostrando alteraciones en el estado de bienestar de los estudiantes, en los datos analizados del presente estudio se encontró una mayor repercusión en el sexo femenino, al igual que en otras investigaciones citadas, dado que enfermería hasta el momento es una profesión con una alta población del sexo femenino.

En el artículo de la Escuela de Enfermería, Facultad de Medicina, Pontificia Universidad Católica de Chile ${ }^{12}$ menciona que un malestar psicológico se asocia significativamente con situaciones altamente estresantes que enfrentan los estudiantes de enfermería durante su experiencia clínica, tales como: contacto con el dolor, sufrimiento, muerte, vivir situaciones urgentes y graves para las que muchas veces no se sienten preparados y miedo a cometer errores, entre otras, comentarios similares se encuentran en la tesis doctoral de Córdoba ${ }^{3}$.

En la escala GQH-12 se encontró un bajo puntaje, parecido como se muestran en nuestros resultados, la mayoría de los estudiantes encuestados presentan bienestar psicológico, al igual que en otros estudios ${ }^{12-14}$. Para la escala PANAS, se coincide con las puntuaciones obtenidas por los autores referenciados siendo el afecto negativo mayor que el efecto positivo, a medida que disminuye NA (afecto negativo) y aumenta PA (afecto positivo) implica una mejor percepción del bienestar psicológico propio.

En la escala ECE se obtienen resultados aproximados, en manifestar cansancio emocional, satisfacción con los estudios y autoestima. En el presente estudio se puede observar como los estudiantes tienen mejor bienestar psicológico, cuanto mayor es la puntuación de Autoestima y Satisfacción con los estudios. En la escala MBI-SS los resultados se muestran en general muy parecidos ya que el valor de la dimensión eficacia académica es el valor más alto, cinismo y agotamiento son los más bajos. Los resultados que muestran en la escala UWES-S, son parecidos en ser inferiores en la puntuación total, UWES-S añade valor a la predicción de indicadores de bienestar psicológico subjetivo, lo que significa que los individuos que manifiestan mejor bienestar psicológico también muestran niveles más elevados en compromiso, vigor y más bajo en absorción.

A partir de la idea que la profesión de enfermería tiene una alta demanda emocional, se puede observar cómo los estudiantes obtienen mejor bienestar psicológico, cuanto mayor es la puntuación de Autoestima y Satisfacción con los estudios, lo que significa que los individuos que manifiestan mejor bienestar psicológico también muestran niveles más elevados en compromiso, vigor y más bajo en absorción. Con todo lo dicho anteriormente, se considera que si se quiere lograr la formación de un profesional integral en Enfermería con el nivel de madurez 
que contemplan en el plan de estudios es necesario tomar en cuenta además de su desarrollo intelectual, el desarrollo de su IE.

La enseñanza de ambas carreras solo se ha centrado en el proceso de diferentes competencias, habilidades y aptitudes enfocadas a desarrollar la parte clínica, por medio del uso de la medición del aprendizaje a través de exámenes, tareas y trabajos sin prepararlos para afrontar situaciones diarias que involucra lo emocional, la percepción de estrés, trabajo en equipo y adaptarse a un espacio cambiante. Se observa que la participación del estudiante en el proceso enseñanza-aprendizaje es por obligación, teniendo en cuenta que el proceso de aprendizaje no solo depende del conocimiento y la capacidad intelectual, sino también de como el estudiante conceptualiza sus emociones para el beneficio propio, actualmente hay que considerar que la IE es también un parámetro útil y necesario que mide la capacidad del individuo de sentir, entender y controlar los estados anímicos propios y de las personas que nos rodean. Se reconoce que la IE es fundamental para la formación académica de los estudiantes de pregrado en enfermería ya que ejercen una función humanista cuyos componentes se basan en los vínculos, responsabilidad, conciencia, conocimiento de sí mismo, capacidad para enfrentar situaciones de estrés

Se reconoce que la IE es fundamental para la formación académica de los estudiantes de pregrado en enfermería ya que ejercen una función humanista cuyos componentes se basan en los vínculos, responsabilidad, conciencia, conocimiento de sí mismo, capacidad para enfrentar situaciones de estrés y la habilidad social que permite la interacción con otros, como aspecto fundamental de la persona. y la habilidad social que permite la interacción con otros, como aspecto fundamental de la persona.

Goleman ${ }^{9}$ menciona que los estudiantes con mejores habilidades y destrezas, tiene mayor capacidad de motivarse, así mismo, de perseverar en el empeño a pesar de las frustraciones, de controlar los impulsos, diferir las gratificaciones, regular los propios estados de ánimo, controlar la angustia, empatizar y confiar en los demás, factores mucho más determinantes para la consecución de una vida plena que las medidas del desempeño cognitivo.

Teniendo como referencia las " 10 habilidades para la vida que propone la OMS"22, menciona que las personas, como seres sociales, se ven obligadas a establecer relaciones con sus semejantes, ya sea en el entorno familiar, educativo, laboral o de ocio. Sin embargo, no siempre es fácil desenvolverse en un determinado contexto social y no es debido a la falta de capacidades, sino porque no se han adquirido las habilidades necesarias para hacerlo. Al ingresar a las carreras mencionadas es un mundo desconocido en el que el estudiante se tiene que enfrentar a ritmos, responsabilidades intensas, además no siempre tienen la fuerza y adecuada IE para afrontar los momentos más difíciles durante su formación académica ${ }^{7}$, sin olvidar que los profesores juegan un papel fundamental en la vida académica del alumno ya que ellos son una parte importante en la formación de nuevos conocimientos; a su vez son el medio para alcanzar los objetivos académicos, reforzar valores, desarrollo de conciencia crítica y reflexiva, pero principalmente, fomentar una actitud propositiva a fin que los estudiantes tengan una plena conciencia de los distintos hechos que sucedan consigo mismo. Sin embargo, algunos cuentan con excelentes conocimientos dentro de su área de estudio, pero no todos con las habilidades pedagógicas para establecer y conservar relaciones interpersonales significativas, necesarias para tener una relación de confianza entre profesor y alumno ${ }^{7-14}$. 
Este ha sido uno de los problemas que no ha tomado en cuenta la universidad como algo que influye en la salud y en la formación académica del estudiante de pregrado en enfermería, ya que el perfil del estudiante responde a una estructura y diseño como un ser maduro, responsable e independiente haciendo a un lado el estado emocional de los universitarios. Al ver esta situación enfermería resulta ser una carrera que posee una alta demanda emocional, propia del trabajo con pacientes críticos, enfermos y en ocasiones altamente demandantes siendo la forma de enfrentar dichas situaciones independiente de las características de personalidad que posee el estudiante ${ }^{12}$. Se puede observar, que es poca la población estudiantil que de manera personal encuentran vías para el desarrollo de la IE y eliminar situaciones de tensión y/o estrés. Por tal motivo, es importante realizar estrategias en relación con la IE para el beneficio del estudiante de enfermería durante su formación académica, con fin de generar una mayor conciencia y fomentar la IE en las aulas y la necesidad de integrar en el curriculum el desarrollo de las habilidades y destrezas en IE, para mejorar el desempeño académico del estudiante de pregrado en enfermería. Recordando que la IE es un concepto clave para organizar las destrezas interpersonales y de comunicación en el ámbito de la profesión en enfermería. Por otro lado la exposición mantenida a factores estresantes en los estudiantes puede producir deterioro del funcionamiento normal del organismo generando, entre otros falta de concentración, dificultad para memorizar, para resolver problemas, déficits en las habilidades de estudio, escasa productividad y un menor rendimiento académico, "diversos estudios epidemiológicos y sociales han demostrado consistentemente que el estrés psicosocial asociado a condiciones adversas de vida se relaciona con un aumento de trastornos mentales, particularmente ansiedad y depresión, además constituye un factor de riesgo para los trastornos por abuso y dependencia a sustancias adictivas" 12 .

Así mismo se ha considerado importante seguir realizando estudios con estudiantes de pregrado de enfermería, desde el comienzo de su carrera hasta su culminación, para medir cuánto han desarrollado su IE y cómo la ponen en práctica en la resolución de problemas básicos de la profesión durante su formación académica, práctica clínica, servicio social y en el futuro mediato del ámbito laboral.

Esto permitirá potencializar a la profesión y brindar cuidados de calidad, de aquí la importancia de considerar la IE como una asignatura pendiente para los estudiantes de pregrado en enfermería, para fomentar la habilidad de saber cómo actuar y/o manejarse emocionalmente en ambos casos, incorporar la IE durante la formación académica es adquirir una competencia social clave que permite relacionarse con los demás, así mismo realizar talleres presenciales que implementen estrategias en el desarrollo de habilidades y destrezas en IE, para que el alumno pueda desarrollar una conciencia que le permita identificar sus propias emociones, el cual le ayudara a incrementar el rendimiento académico, bienestar y equilibrio social, disminuyendo en la deserción de la carrera, enfermedades, consumo de sustancias adictivas y surgimiento de conductas disruptivas. Se sabe que desde la praxis cotidiana, una actuación inteligente es saber identificar, actuar y conocer nuestras propias emociones esto permite poder actuar de manera reflexiva, estableciendo relaciones adecuadas entre los pensamientos, las emociones y el comportamiento.

\section{Conclusiones}

Una vez analizados los resultados obtenidos, podemos decir que se logró conocer la IE de los estudiantes de la profesión de enfermería en el pregrado cuando realizan las prácticas clínicas y comunitarias en los diferentes espacios existentes para ello y tramitados por la institución 
educativa. Es evidente que todos hemos escuchado hablar de emociones y experimentarlas pero son muy pocos quienes saben reconocerlas y actuar de acuerdo a la situación en la que se presentan. Los estudiantes de la LE y la LEO obtienen una preparación teórica-práctica en cuanto habilidades y destrezas para el desarrollo profesional. Sin embargo, no se les prepara para afrontar situaciones diarias que involucra lo emocional, se observa que su participación en el proceso enseñanza-aprendizaje es por obligación. Se reconoce que la IE es fundamental para la formación académica de los estudiantes de pregrado en enfermería ya que ejercen una función humanista cuyos componentes se basa en la empatía, responsabilidad, conciencia, conocimiento de sí mismo, capacidad para enfrentar situaciones de estrés y la habilidad social que permite la interacción entre otros, como aspecto fundamental de la persona.

Es necesario que las autoridades de las escuelas y facultades de enfermería, así como los profesionales de enfermería de las instituciones de salud le den importancia a la IE para que den las facilidades que permitan iniciar cursos o talleres en donde se capacite de cómo se puede hacer el cuidado de enfermería a la población con IE de los estudiantes de enfermería.

Conflictos de interes: Los autores declaran no tener conflicto de interés.

Financiamiento: Escuela Nacional de Enfermería y Obstetricia de la Universidad Nacional Autónoma de México

\section{Referencias}

1. Escuela Nacional de Enfermería y Obstetricia; Universidad Nacional Autónoma de México; Plan de estudios de la licenciatura en enfermería; aprobado por el H. Consejo Técnico el 9 de diciembre del 2008, pág. 45.

2. Liébana PC. Salud emocional en estudiantes universitarios de enfermería y fisioterapia de Castilla y León [Tesis doctoral]. Córdoba: Universidad de Córdoba; 2012. Disponible en: https://helvia.uco.es/xmlui/handle/10396/8088

3. Ruiz M. Castellanos, E, \& Enríquez, C. Factores que favorecen el desarrollo de inteligencia emocional y su impacto en el rendimiento académico del estudiante universitario. Revista Iberoamericana para la Investigación y el Desarrollo Educativo. 2013;10: Disponible en; https:// studylib.es/doc/4435024/factores-que-favorecen-el-desarrollo-de-inteligencia-emoc

4. Vallés A, Vallés C. Inteligencia Emocional. Aplicaciones educativas. España: Editorial EOS; 2000. p. 20-22

5. Hernández VC, Dickinson BM. Importancia de la inteligencia emocional en Medicina. Inv. Ed Med. 2014;3(11):155-160. https://doi.org/10.1016/S2007-5057(14)72742-5

6. Extremera PN \& Ruiz AD. Inteligencia emocional y bienestar personal en estudiantes universitarios de ciencias de la salud. (Tesis doctoral). Departamento de psicología básica. Universidad de Málaga. 2012. Disponible en: https://riuma.uma.es/xmlui/bitstream/handle/10630/5384/TDR_PINEDA_GALAN.pdf?sequence=1

7. Sánchez NT, Humé FM. Evaluación e interpretación en Inteligencia emocional y su importancia en el ámbito educativo. Universidad de Castilla-La Mancha. Toledo. España. 2004 Disponible en: https://ruidera.uclm.es/xmlui/bitstream/handle/10578/7972/Evaluaci_n_e_intervenci_n_en_inteligencia_emociona.pdf?sequence $=1$

8. Sánchez G. Las emociones en la práctica enfermera [Tesis doctoral]. Barcelona: Universidad Autónoma de Barcelona; 2014. Disponible en: https://ddd.uab.cat/record/127628

9. Goleman D. La naturaleza de la inteligencia emocional. En: Best-seller mundial. Colección ensayo. Inteligencia emocional.62 ed. Barcelona: kairós; 2006. P 25-75 
10.Prieto D, Inciarte J, Rincón C \& Bonilla E. Estudio del coeficiente emocional en estudiantes de medicina. Revista chilena de neuro-psiquiatría. 2008;46(1):10-15. https://doi.org/10.4067/S0717-92272008000100002

11.Herrera TL, Mohamed ML, Cepero ES. Cansancio Emocional en estudiantes universitarios. DEDiCA. Revista de Educação e Humanidades.. 2016;9:173-191. https://dialnet.unirioja.es/descarga/articulo/5429381.pdf

12. Herrera LM, Rivera MS. Prevalencia de malestar psicológico en estudiantes de enfermería relacionada con factores sociodemográficos, académicos y familiares. Ciencia y Enfermería. 2011;2:55-64. https://doi.org/10.4067/S0717-95532011000200007

13.Ríos EM, Moncada AL, Llanos RG, Satana GR Salinas GH. Perfil psicológico de los estudiantes de $1^{\circ}$ año de enfermería. Estudio preliminar. Ciencia y enfermería. 2009;15(1):99-108 http://dx.doi.org/10.4067/S0717-95532009000100011

14.Alves AJ, Alves RM, Pineda DJ. Evaluación de los estados emocionales de estudiantes de enfermería. Índex de Enfermería. 2007;16(56):1132-1296.

https://doi.org/10.4321/S1132-12962007000100006

15.Cortes JF, Barragán C, Vázquez CM. Perfil de inteligencia emocional: construcción, validez y confiabilidad]. Salud Mental. 2002;25(5):50-60. Disponible en: http://www.redalyc.org/pdf/582/58252506.pdf

16.Campo-Arias A. Cuestionario general de salud-12: análisis de factores en población general de Bucaramanga, Colombia. Iatreia . 2007;20(1):29-36. Disponible en: http://www.scielo.org. co/scielo.php?script=sci_arttext\&pid=S0121-07932007000100004\&lng=en.

17. Molina MB, Salazar VM. Escala de Afectividad (PANAS) de Watson y Clark en Adultos Mayores. Compendio de instrumentos de Medición IIP-2014 [consultado 18 de ago 2017]. Smith-Castro. Universidad de Costa Rica. 2014. Disponible en: http://www.kerwa.ucr.ac.cr/ bitstream/handle/10669/30348/Escala\%20de\%20Afectividad\%20\%20(PANAS)\%20de\%20 Watson\%20y\%20Clark\%20en\%20Adultos\%20Mayores.PDF?sequence $=4$

18. Hederich MC, Caballero DC. Validación del cuestionario Maslach Burnout Inventory-Student Survey (MBI-SS) en contexto académico colombiano. Revista CES Psicología [Internet] 2016;9(1). https://doi.org/10.21615/cesp.9.1.1

19.Liébana PE, Fernández ME, Morán AC. Relación entre la inteligencia emocional y el burnout en estudiantes de enfermería. Psychology, Society, \& Education 2017;9(3):335-345 Doi https://doi.org/10.25115/psye.v9i3.856

20.Graue WE, Álvarez CR, Sánchez MM. El Síndrome de "Burnout": La despersonalización, el agotamiento emocional y la insatisfacción en el trabajo como problemas en el ejercicio de la medicina y el desarrollo profesional. [Internet]. Seminario El Ejercicio Actual de la Medicina: FACMED-UNAM [consultado 18 de ago 2017]. Disponible en:

http://www.facmed.unam.mx/sms/seam2k1/2007/jun_01_ponencia.html

21.Medrano LA, Moretti L, Ortiz A. Medición del Engagement Académico en Estudiantes Universitarios. Revista Iberoamericana de Diagnóstico y Evaluación - e Avaliação Psicológica. 2014;2(40):114-123 disponible en: https://www.redalyc.org/pdf/4596/459645432012.pdf

22.Arboix M. Consumer.Fundacion EROSKI. Psicología. Salud mental [internet] 16 sep. 2016. Las 10 habilidades para la vida. [Consultado: 28 ago. 2018]. Disponible en: http://www.consumer.es/web/es/salud/psicologia/2016/09/16/224273.php 